\title{
Single-center experience demonstrating low adverse events and high efficacy with self-expandable metal esophageal and biliary stents for pseudocyst and walled off necrosis drainage
}

\section{(ㄷ)(우우}

\author{
Authors \\ Ronald Dungca Ortizo, Farid Jalali, Daniel Thieu, Allen Yu, Robert Bucayu, Christopher Paiji, Kyle Fortinsky, Kenneth \\ Chang, John Gunn Lee, Jason Buddika Samarasena
}

Institution

H. H. Chao Comprehensive Digestive Disease Center, University of California, Irvine Medical Center, Orange, California, United States

submitted 11.2.2019

accepted after revision 30.9.2019

\author{
Bibliography \\ DOI https://doi.org/10.1055/a-1178-0185 | \\ Endoscopy International Open 2020; 08: E1156-E1160 \\ (c) Georg Thieme Verlag KG Stuttgart · New York \\ elSSN 2196-9736
}

\section{Corresponding author}

Jason B. Samarasena MD, FACG, Associate Professor of Medicine, Division of Gastroenterology and Hepatology, H. H. Chao Comprehensive Digestive Center, University of California, Irvine Medical Center, 101 The City Drive South, Orange, CA 92868

Fax: +1-714-456-7520

jsamaras@uci.edu

\section{ABSTRACT}

Background and study aims Lumen-apposing metal stents (LAMS) have been designed as proprietary stents for the management of pseudocysts (PC)/walled off necrosis (WON). There has been concern about adverse events
(AEs) with LAMS including bleeding, buried stent syndrome and migration. Prior to LAMS becoming available, fully-covered self-expandable metal esophageal and biliary stents (FCSEMSs) were used off-label for management of PC/ WON with many centers demonstrating low rates of AEs. The primary aim of this study was to study the safety and efficacy of FCSEMS for the management of pseudocysts/ WON.

Patients and methods This was a retrospective review of all endoscopic ultrasound (EUS)-guided placement of FCSEMSs for drainage of PC/WON cases performed at our institution over 4-year period. The primary outcomes studied were technical success, AEs, PC/WON resolution, and salvage surgical/radiologic intervention.

Results Technical success achieved in 65 of $65(100 \%)$ study patients. An AE occurred 0 of 25 patients ( $0 \%$ with PC, and in 10 of 40 patients ( $25 \%$ ) with WON: bleeding (3\%), migration (5\%) and stent dysfunction/infection (18\%). There was resolution in 25 of 25 patients $(100 \%)$ with a PC and 31 of 40 patients (78\%) with a WON. Salvage therapy by interventional radiology or surgery was performed in nine of 40 patients (22\%).

Conclusions This single-center 4-year experience in the pre-LAMS era showed that FCSEMS was safe and effective in all patients with PC and over $75 \%$ of patients with WON. Given the large cost differential between LAMS and FCSEMS and the efficacy and safety shown with FCSEMS, we believe that FCSEMS should still be considered a first-line option for patients with pancreatic fluid collections, particularly in patients with PCs.

\section{Introduction}

Pancreatic fluid collections (PFCs) such as pseudocysts (PC) and walled-off pancreatic necrosis (WON) result due to acute pancreatitis, chronic pancreatitis, or trauma. Conservative management of PFCs is usually indicated in patients with minimal or no symptoms whereas patients who are symptomatic require drainage. Surgical drainage techniques are associated with significant morbidity and mortality [1-3]. More recently, endo- scopic drainage via transmural stents has emerged as the mainstay of treatment due to their efficacy and improved safety profile. Studies have reported an $84 \%$ to $94 \%$ technical success rate for endoscopic-guided PFC drainage [4-8].

Current endoscopic drainage techniques allow for placement of stents via direct real-time endoscopic ultrasound (EUS) guidance. The two main types of stents used for drainage include fully-covered self-expanding metal stents (FCSEMS) and lumen-apposing metal stents (LAMS). Current data are conflict- 
ing as to which stent performs best in a given patient with a PFC. Furthermore, the stent being used may differ depending on whether the goal is to drain a PC versus a WON.

Walter et al examined the use of LAMS for drainage of a PC versus a WON. This study showed a $93 \%$ resolution rate in PC and $81 \%$ in WON, with $7 \%$ adverse events (AEs) in PC and $9 \%$ in WON [9]. A similar study performed by Chandran et al showed LAMS to be slightly less effective with higher rates of AEs. Their study showed $76 \%$ of the PCs resolved versus $66 \%$ of the WON, with $38 \%$ AEs in PC and $33 \%$ AEs rates in WON [10].

Sharaiha et al performed a study on FCSEMS for PC, which demonstrated a higher resolution rate of $98 \%$ with an AE rate of $16 \%$ [11]. In 2016, Siddiqui et al performed a retrospective study on LAMS, which had a $100 \%$ resolution rate for PC and $88.2 \%$ for WON [12]. Siddiqui et al performed another retrospective study in 2017 comparing LAMS vs FCSEMS for drainage of WON. This study demonstrated a $95 \%$ resolution for FCSEMS compared to $90 \%$ for LAMS. In addition, FCSEMS had lower overall rates of AEs when compared to LAMS [13].

Currently, the American Society for Gastrointestinal Endoscopy (ASGE) guidelines recommend drainage of symptomatic, rapidly enlarging, or infected PFC. There are no current guideline recommendations as to which type of stent is preferred for the management of a PC or WON [14]. With the advent of LAMS, there has been a trend nationally to place LAMS routinely and indiscrimantly in all PFCs. We present a single-center experience demonstrating low AE rates and high efficacy with self-expandable metal esophageal and biliary stents for PC and WON drainage.

\section{Patients and methods}

This was a retrospective study performed at the University of California, Irvine Medical Center (UCIMC) on consecutive patients receiving EUS-guided PC or WON drainage over a 4-year period between November 2012 and September 2016. There was no availability of LAMS at our institution during the study period. All cases were identified through the institution's electronic medical record (EMR). This study was approved by our institution's IRB.

\section{Inclusion criteria}

Consecutive patients with symptomatic PC or WON who underwent endoscopic drainage using EUS-guided placement of a FCSEMS at our institution were included. Based on imaging, a PFC without any internal debris was considered a PC, and a PFC with debris inside was considered a WON. Patients required at least one follow-up visit at our institution with or without follow-up imaging.

\section{Definitions of outcomes}

The primary outcomes measured in this study were resolution of PFC and AEs. Resolution was defined as complete improvement of symptoms and $\geq 50 \%$ decrease in PFC size on repeat imaging if available. An AE was defined as any procedural-related complication during study follow-up.
Secondary outcomes included technical success and failure of endoscopic therapy. Technical success was defined as successful placement of the FCSEMS at the time of the procedure. Failure of endoscopic therapy was defined as a patient remaining symptomatic despite FCSEMS stent placement and requiring repeat endoscopic intervention (not only for stent removal) or salvage therapy with either surgical or IR percutaneous drainage.

\section{Data collected}

The following data were extracted from the EMR at UCIMC: patient demographic information (age and gender), etiology of pancreatitis, size of PFC (determined by EUS, MRCP or CT), type of PFC (PC or WON), type of stent placed (FCSEMS), size of stent placed, complications of stent placement (stent dysfunction/infection, bleeding, migration, perforation/pneumoperitoneum), follow-up data, and requirement for salvage therapy (endoscopic, surgical or interventional radiology [IR] drainage). The approximate costs related to the endoscopic care of each patient were calculated. Multiple variables were included in this cost evaluation including the retail price of the FCSEMS cost, additional procedural accessories, procedural stent placement cost, procedural stent removal cost, and cost related to debridement procedures and stent replacement.

\section{EUS-guided PFC drainage procedures}

Each procedure was performed by one of two endoscopists (JS or JL) with extensive experience in endoscopic ultrasound (EUS) and therapeutic endoscopy. Procedures were performed with patients under general anesthesia. The endoscope was advanced into the stomach or duodenum, with continuous EUS imaging for PFC characterization. Once the optimal entry point was identified, a needle with stylet was inserted into the PFC under real-time EUS guidance. Per protocol, $10 \mathrm{~mL}$ of negative pressure was applied for aspiration of the PFC. A guidewire was then passed through the needle and coiled inside the PFC. A needle-type knife or tapered catheter was passed over the guidewire. Using electrocautery or with dilation alone, a fistula was created between the stomach or duodenum and the PFC. This fistula was then dilated with a balloon to allow for stent insertion. A FCSEMS was advanced over the guidewire and deployed between the PFC and the gastric or duodenal lumen ( $\triangleright$ Fig.1). There were two types of stents used based on the preference of the endoscopist (Conmed viabil biliary stent or Taewoong esophageal stent). Additional procedures were performed as needed for patients not responding to initial drainage. During additional procedures, the endoscopist determined the best course of action which may have included placement of a pigtail stent or introduction of a slim endoscope through the FCSEMS to perform debridement of the PFC. Challenging cases refractory to endoscopic management were discussed with interventional radiologists and surgical teams to consider all potential management options. 

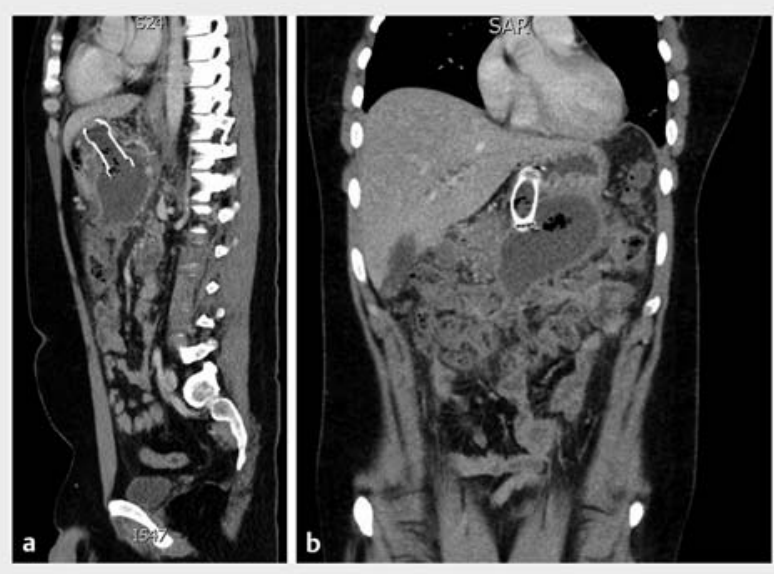

- Fig. 1 CT image of FCSEMS transgastric within WON. a Sagittal view. $\mathbf{b}$ Coronal view.

\section{Patient follow-up}

After endoscopic drainage, patients were followed up in clinic sometimes with repeat cross-sectional or EUS imaging. If the patient was asymptomatic and the PFC resolved on imaging, the stent was routinely removed. If the patient reported ongoing symptoms, repeat imaging and/or endoscopy was performed for further evaluation and additional procedures were performed as required. Patients were followed up until complete resolution of symptoms.

\section{Results}

A total of 65 patients were included in the final analysis ( $\triangleright \mathrm{Ta}$ ble 1). Technical success of FCSEMS placement was achieved in 65 of 65 patients (100\%). AEs occurred in none of 25 patients $(0 \%)$ with a PC, and 10 of 40 patients $(25 \%)$ with WON ( $\triangleright$ Table 2). There were no serious AEs. In one patient there was bleeding after the initial placement that was self-limited and required no intervention. In two patients the stent had migrated on follow-up imaging without clinical consequence and in both instances new stents were placed. In seven patients there was evidence of stent dysfunction/occlusion with infection deemed likely related to this dysfunction/occlusion. In three of these cases, a $10-\mathrm{mm}$ diameter biliary stent was replaced by a larger 20-mm diameter esophageal stent.

\section{Resolution and salvage therapy}

There was resolution in 25 of 25 patients (100\%) with a PC and 31 of 40 patients $(78 \%)$ with WON. Of the patients with WON, nine of $40(22 \%)$ required interventional radiology or surgical intervention while none of 25 patients ( $0 \%$ ) with a $P C$ required IR or surgery.

\section{Debridement and/or additional stenting}

None of 25 patients ( $0 \%$ ) with a PC who needed an additional stent to achieve resolution compared to 30 of 40 patients (75\%) with a WON who required at least one more additional
- Table 1 Baseline characteristics of study patients.

\begin{tabular}{|c|c|c|}
\hline Characteristic & $P C(N=25)$ & WON $(N=40)$ \\
\hline Gender & $18(72 \%)$ & $30(75 \%)$ \\
\hline Female & $7(28 \%)$ & $10(25 \%)$ \\
\hline Mean age (years) & $50.2(15-18)$ & $53.8(13-90)$ \\
\hline \multicolumn{3}{|l|}{ Etiology of pancreatitis } \\
\hline Gallstone & $9(36 \%)$ & $16(40 \%)$ \\
\hline Alcohol & $7(28 \%)$ & $3(7 \%)$ \\
\hline $\begin{array}{l}\text { Other (medications/autoim- } \\
\text { mune) }\end{array}$ & $4(16 \%)$ & $10(25 \%)$ \\
\hline Unknown & $5(20 \%)$ & $11(28 \%)$ \\
\hline $\begin{array}{l}\text { Mean size of } \mathrm{PFC}(\mathrm{mm}) \pm \mathrm{SD} \\
\text { (Range) }\end{array}$ & $\begin{array}{l}92.7 \pm 33.5 \\
(42-170)\end{array}$ & $\begin{array}{l}118.4 \pm 59.8 \\
(22-290)\end{array}$ \\
\hline \multicolumn{3}{|l|}{ Location of PFC drainage } \\
\hline Transgastric & $24(96 \%)$ & $40(100 \%)$ \\
\hline Transduodenal & $1(4 \%)$ & $0(0 \%)$ \\
\hline \multicolumn{3}{|l|}{ Location of PFC } \\
\hline Head/neck & $2(8 \%)$ & $3(8 \%)$ \\
\hline Body/tail & $15(60 \%)$ & $21(52 \%)$ \\
\hline Other/not reported & $8(32 \%)$ & $16(40 \%)$ \\
\hline \multicolumn{3}{|c|}{$\begin{array}{l}\text { PC, pseudocyst; WON, wall off necrosis; PFC, pancreatic fluid collection; } \\
\text { SD, standard deviation }\end{array}$} \\
\hline
\end{tabular}

- Table 2 Technical success and adverse events.

\begin{tabular}{|l|l|l|}
\hline Outcome & PC (N=25) & WON (N=40) \\
\hline Technical success & $25(100 \%)$ & $40(100 \%)$ \\
\hline Adverse events & & \\
\hline Bleeding & $0(0 \%)$ & $1(3 \%)$ \\
\hline Migration & $0(0 \%)$ & $2(5 \%)$ \\
\hline Stent dysfunction/infection & $0(0 \%)$ & $7(18 \%)$ \\
\hline Perforation/pneumoperitoneum & $0(0 \%)$ & 0 \\
\hline PC, pseudocyst; wON, walled off necrosis & \\
\hline
\end{tabular}

stent. There were none of 25 patients ( $0 \%)$ with a PC who required debridement, compared to 27 of 40 patients (67.5\%) with a WON who required at least one debridement. Patients with a $\mathrm{PC}$ required on average 1.8 procedures per patient, while patients with a WON required on average 3.9 procedures per patient. In cases where debridement needed to be performed, often the initially placed FCSEMS was removed, the debridement was performed and a new stent was placed during the same session. In the 40 patients with WON, a total of 79 stents were used in their treatment ( $\mathbf{F i g . 2}$ ). 


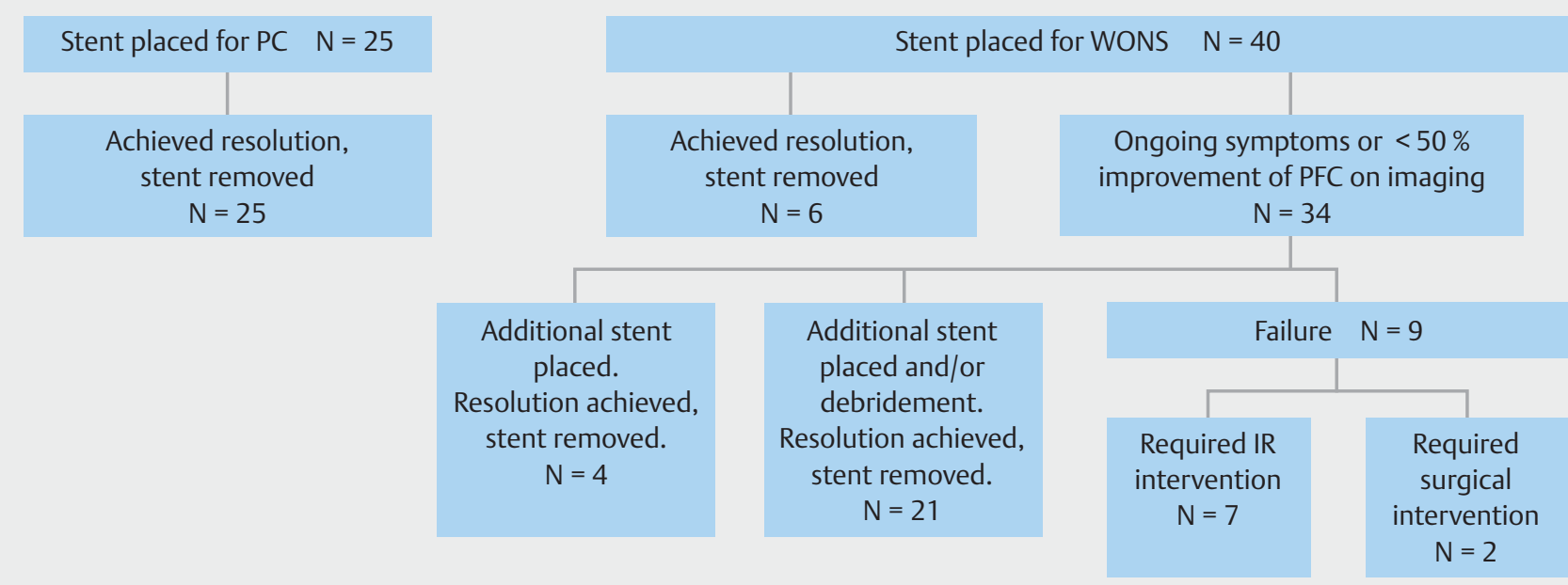

Fig. 2 Flowchart summary of procedures required for patients undergoing stent therapy with PC and WON.

\section{Discussion}

The choice of stent for EUS-guided drainage of symptomatic PFCs remains controversial and depends on a number of factors. Current guidelines from ASGE highlight indications for drainage but are non-definitive when it comes to choice of stent [14]. Different studies have used various techniques in achieving resolution with varying degrees of success. The current study adds to the growing body of literature suggesting that FCSEMS are effective and safe in the management of patients with PC.

Previous studies have compared plastic and metal stents in the management of PCs. In 2014, Lee et al performed a randomized, single-blind prospective study that demonstrated equal efficacy between metal and plastic stents for drainage of PC. In this study, 52 patients with peripancreatic fluid collections were entered into the study (with 2 patients being excluded due to exclusion criteria). These patients were then randomly assigned to receive either a FCSEMS or plastic stents for EUSguided drainage of the peripancreatic fluid collections. It was concluded that EUS-gided PFC drainage with FCSEMS and plastic stents are comparable to each other with regards to safety, efficacy and technical success [15]. Sharaiha et al performed a retrospective study in 2015. This study included 230 patients with pancreatic pseudocysts who had EUS-guided drainage, with 118 patients having double-pigtail (DP) stents and 112 patients having FCSEMS. At 12-month follow-up, it was found that only $89 \%$ of patients with a DP stent had complete resolution compared to $98 \%$ of patients with a FCSEMS which was found to be statistically significant with $P=0.01$. This concluded better success rates and less AEs for FCSEMS versus plastic stents when managing PCs [11].

There have been studies evaluating the safety and efficacy of LAMS with regard to PC and WON. In 2015, Walter et al performed a prospective study which concluded that LAMS had effective clinical success. There was a $93 \%$ success rate in PCs and $81 \%$ in WON [9]. On the other hand, Chandran et al performed a study in 2015 that showed compromised resolution rates and higher rates of AEs. Here, only $76 \%$ of PCs and $66 \%$ of WON resolved, with $38 \%$ of PCs and $33 \%$ of WONs having AEs [10]. In addition, Siddiqui et al performed a retrospective cohort study in 2017 which compared outcomes and AEs of EUS-guided drainage and debridement of WON with DP stents, FCSEMS and LAMS. A total of 313 patients were included in the study, with 106 using DP stents, 121 using FCSEMS and 86 using LAMS. When comparing the three groups, early AEs were significantly lower in the FCSEMS when compared with the DP and LAMS groups $(1.6 \%, 7.5 \%$, and $9.3 \% ; P<0.1)$. At 6 -month follow-up, resolution of WON was lower in DP stents when compared to FCSEMS and LAMS ( $81 \%$ vs $95 \%$ vs $90 \% ; P=0.001)$. The mean number of procedures required for resolution of WON was lower in the LAMS group when compared with FCSEMS and DP ( 2.2 vs 3 vs 3.6; $P=0.04$ ). Overall, this study by Siddiqui et al concluded that EUS-guided drainage/debridement of WON using LAMS and FCSEMS is superior to DP stents with regards to efficacy [13].

Studies that have evaluated FCSEMS with regard to their safety and efficacy when managing both PC and WON are limited. In 2016, a large Spanish retrospective noncomparative review performed by Vasquez-Sequeiros et al which had 211 patients (53\% PC, $47 \%$ WON) demonstrated that technical success was achieved in $97 \%$ of patients, AEs occurring in $21 \%$ of patients, short-term clinical effectiveness 2 weeks after the intervention) achieved in $94 \%$ of the patients, and long-term clinical effectiveness ( 6 months after the intervention) achieved in $85 \%$ of the patients. This study concluded that FCSEMS is safe and effective for drainage of PC and WON [16].

There is a significant difference in cost between FCSEMS and LAMS. The cost of LAMS is approximately $\$ 5,300$, and FCSEMS cost approximately $\$ 1,800$. Our retrospective study supports the belief that using a FCSEMS in comparison to a LAMS for management of a PC and WON would contribute to decreased costs for the patient and hospital. The estimated total cost to treat all 65 patients in our study was $\$ 409,600$. In a cost model 
where patients with both a PC or a WON receive a LAMS to treat the same 65 patients the projected cost is $\$ 544,500$ and this is with the assumption that there were no LAMS placement failures and all PFC resolved after one LAMS placement. With less favorable assumptions, the cost differential could be significantly more.

The current study revealed that FCSEMS was safe in patients with PC with all patients acheieving resolution of their PFC without any AEs. In over $75 \%$ of patients with WON, there were no AEs with placement of FCSEMS. Given the large cost differential between LAMS and FCSEMS and the efficacy and safety shown with FCSEMS, we believe that FCSEMS should be considered a reasonable first-line option for patients with PFCs, particularly in patients with pseudocysts. One design aspect that potentially could make FCSEMS safer than the current LAMS stent available in the United States is the lack of the wide flanges that allow the FCSEMS to migrate or slide out toward the lumen when a PC or WON is getting smaller over time. As the collection gets smaller and the wall of the collection abuts the distal aspect of the stent, a LAMS will not move and the edges of the stent can potentially irritate and induce friction with the wall of the collection. A FCSEMS has the ability to be "pushed out" of the collection by the wall and therefore the risk of friction with the wall may be lessened. In our series, it was not uncommon to see a FCSEMS to have partially migrated out further into the lumen at the time of stent removal.

Our study has several limitations. First, the study design was retrospective. Despite this, consecutive patients over the study period were included and no patients treated for PFC were excluded. Second, our sample population was a relatively small single-center experience with highly experienced endoscopists. Therefore, it is unknown whether other centers would demonstrate similar efficacy and safety results. Ideally, a prospective, randomized, comparative study is needed to more thoroughly answer the question of which stent (FCSEMS vs LAMS) is better in each type of PFC (PC and WON). Third, time to follow-up was not standardized so it is unclear precisely when patients had resolution of symptoms. For example, the resolution times for PC (5.6 months) vs WON (5.5 months) were very similar. This is likely because several of the patients with a PC did not return for their follow-up appointments until much later, effectively increasing the length of resolution time.

\section{Conclusion}

In conclusion, this single-center 4-year experience in the preLAMS era showed that FCSEMS was safe and effective in all patients with PC and over $75 \%$ of patients with WON. Given the large cost differential between LAMS and FCSEMS and the efficacy and safety shown with FCSEMS, we believe that FCSEMS should still be considered a first-line option for patients with PFCs, particularly in patients with pseudocysts.
Competing interests

J. Samarasena - Consultant: Medtronic, Olympus, Conmed, Neptune medical, Motus, Pentax, Mauna Kea Technologies.

References

[1] Köhler H, Schafmayer A, Lüdtke FE. Surgical treatment of pancreatic pseudocysts. Br J Surg 1987; 74: 813-815

[2] Gumaste VV, Pitchumoni CS. Pancreatic pseudocyst. Gastroenterologist 1996; 4: 33-43

[3] Bergman S, Melvin WS. Operative and nonoperative management of pancreatic pseudocysts. Surg Clin North Am 2007; 87: 1447-1460, ix

[4] Antillon MR, Shah RJ, Stiegmann G. Single-step EUS-guided transmural drainage of simple and complicated pancreatic pseudocysts. Gastrointest Endosc 2006; 63: 797-803

[5] Kahaleh M, Shami VM, Conaway MR et al. Endoscopic ultrasound drainage of pancreatic pseudocyst: a prospective comparison with conventional endoscopic drainage. Endoscopy 2006; 38: 355-359

[6] Krüger M, Schneider AS, Manns MP et al. Endoscopic management of pancreatic pseudocysts or abscesses after an EUS-guided 1-step procedure for initial access. Gastrointest Endosc 2006; 63: 409-416

[7] Varadarajulu S, Lopes TL, Wilcox CM et al. EUS versus surgical cystgastrostomy for management of pancreatic pseudocysts. Gastrointest Endosc 2008; 68: 649-655

[8] Park DH, Lee SS, Moon SH et al. Endoscopic ultrasound-guided versus conventional transmural drainage for pancreatic pseudocysts: a prospective randomized trial. Endoscopy 2009; 41: 842-848

[9] Walter D, Will U, Sanchez-Yague A et al. A novel lumen-apposing metal stent for endoscopic ultrasound-guided drainage of pancreatic fluid collections: a prospective cohort study. Endoscopy 2015; 47: 63-67

[10] Chandran S, Efthymiou M, Kaffes A et al. Management of pancreatic collections with a novel endoscopically placed fully covered self-expandable metal stent: a national experience (with videos). Gastrointest Endosc 2015; 81: 127-135

[11] Sharaiha RZ, DeFilippis EM, Kedia P et al. Metal versus plastic for pancreatic pseudocyst drainage: clinical outcomes and success. Gastrointest Endosc 2015; 82: 822-827

[12] Siddiqui AA, Adler DG, Nieto J et al. EUS-guided drainage of peripancreatic fluid collections and necrosis by using a novel lumen-apposing stent: a large retrospective, multicenter U.S. experience (with videos). Gastrointest Endosc 2016; 83: 699-707

[13] Siddiqui AA, Kowalski TE, Loren DE et al. Fully covered self-expanding metal stents versus lumen-apposing fully covered self-expanding metal stent versus plastic stents for endoscopic drainage of pancreatic walled-off necrosis: clinical outcomes and success. Gastrointest Endosc 2017; 85: 758-765

[14] Muthusamy V, Chandrasekhara V, Acosta RD et al. The role of endoscopy in the diagnosis and treatment of inflammatory pancreatic fluid collections. ASGE Standards of Practice Committee. Gastrointestinal Endoscopy 2016; 83: 481-488

[15] Lee BU, Song T], Lee SS et al. Newly designed, fully covered metal stents for endoscopic ultrasound (EUS)-guided transmural drainage of peripancreatic fluid collections: a prospective randomized study. Endoscopy 2014; 46: 1078-1084

[16] Vazquez-Sequeiros E, Baron TH, Pérez-Miranda M et al. Evaluation of the short- and long-term effectiveness and safety of fully covered self-expandable metal stents for drainage of pancreatic fluid collections: results of a Spanish nationwide registry. Gastrointest Endosc 2016; 84: 450-457.e2 